\title{
Horizontal divergence of typhoon-generated gravity waves in the upper troposphere and lower stratosphere (UTLS) and its influence on typhoon evolution
}

\author{
S. H. Kim ${ }^{1}$, H.-Y. Chun ${ }^{2}$, and W. Jang ${ }^{2}$ \\ ${ }^{1}$ Center of Excellence in Earth Systems Modeling and Observations, Chapman University, Orange, CA, USA \\ ${ }^{2}$ Department of Atmospheric Sciences, Yonsei University, Seoul, Korea \\ Correspondence to: H.-Y. Chun (chunhy@yonsei.ac.kr)
}

Received: 14 February 2013 - Published in Atmos. Chem. Phys. Discuss.: 6 November 2013

Revised: 11 February 2014 - Accepted: 28 February 2014 - Published: 1 April 2014

\begin{abstract}
The characteristics of horizontal divergence induced by typhoon-generated gravity waves (HDTGWs) and the influence of HDTGW on typhoon evolution are investigated based on the simulation results of Typhoon Saomai (2006) using the Weather Research and Forecasting (WRF) model. The power spectral density of HDTGW shows dominant powers at horizontal wavelengths of $20-30 \mathrm{~km}$ and at periods of less than $1 \mathrm{~h}$. This is associated with gravity waves generated by vigorous convective clouds in an inner core region of the typhoon. However, the domain-averaged HDTGW in the upper troposphere and lower stratosphere had a spectral peak at $24 \mathrm{~h}$, which is well correlated with the minimum sea-level pressure of the typhoon, especially during a rapidly developing period. The $24 \mathrm{~h}$ period of the averaged HDTGW stems from the inertia-gravity waves generated by the convective clouds in the spiral rainbands, and showed no clear association with the thermal tides or the diurnal variation of precipitation.
\end{abstract}

\section{Introduction}

A typhoon consists of strong convective clouds having various spatiotemporal scales, which can generate inertiagravity waves (IGWs) that reach to the stratosphere. Several observational (e.g., Dhaka et al., 2003; Chun et al., 2007) and numerical modeling (e.g., Kim et al., 2005; Kuester et al., 2008; Kim and Chun, 2010, 2011) studies have investigated the characteristics of vertically propagating gravity waves generated by the convective clouds associated with ty- phoons. Among these studies, Kim et al. (2005) showed that typhoon-generated gravity waves (TGWs) could be an important source of the momentum budget in the middle atmosphere. Eastward-propagating TGWs in the summer hemisphere that survive into the upper stratosphere and mesosphere in easterly background winds can transport the positive momentum in the summer hemisphere required to maintain the wind and temperature in the middle atmosphere general circulation.

Recently Kim and Chun (2011; KC11 hereafter) investigated the effects of TGWs on the environmental flows of a typhoon by examining changes in the vertical wind shear and horizontal divergence in the upper troposphere and lower stratosphere (UTLS). Based on a simulation of Typhoon Saomai (2006), they showed that TGWs contribute significantly to the upper-level horizontal divergence near the typhoon center, with relatively less contribution to the vertical wind shear. The effects of upper-level divergence on the evolution of typhoons have been investigated in several previous studies (e.g., Merrill, 1988; Bosart et al., 2000; Rappin et al., 2011). Among these, Rappin et al. (2011) showed that a typhoon can be intensified by reducing resistance of the upper-level outflow associated with the secondary circulation of typhoon convection based on the Carnot theory of the maximum potential intensity (Emanuel, 1986). They suggested that typhoon outflow weakens the environmental inertial stability, even under the upper-level jet, which minimizes the energy expended in developing and expanding the outflow, and allows for more energy to be utilized for typhoon intensification. 
Considering that horizontal divergence in the upper troposphere is one of the major dynamic factors determining surface pressure tendency (Holton, 1992), and that TGWs contribute significantly to horizontal divergence during typhoon evolution, there may be feedback between TGWs and their sources. The objective of this study is to understand this feedback process. To do so, we examine the characteristics of the horizontal divergence of typhoon-generated gravity waves (HDTGWs) in the UTLS, and their contribution to typhoon evolution based on the Weather Research and Forecasting (WRF) model-simulated results of Typhoon Saomai (2006) that was reported by Kim and Chun (2010; KC10 hereafter).

\section{Numerical experiment}

The numerical model used for this study is the Advanced Research Weather Research and Forecasting (WRF-ARW) model version 2.2. The simulations used three domains (D1, $\mathrm{D} 2$, and D3, hereafter) with horizontal grid spacings of $27 \mathrm{~km}$ (D1), $9 \mathrm{~km}$ (D2), and $3 \mathrm{~km}$ (D3). The number of horizontal grid points is $187 \times 187,253 \times 253$, and $397 \times 397$ for D1, $\mathrm{D} 2$, and D3, respectively. The center of D1 is at $20^{\circ} \mathrm{N}$ and $128^{\circ} \mathrm{E}$. The model employed two-way nesting with an innermost domain (D3) designed to follow the typhoon center. Seventy-seven vertical levels were used from the surface to $5 \mathrm{hPa}(z=\sim 35 \mathrm{~km})$ with a damping layer at the uppermost $5 \mathrm{~km}$. The resultant physical domain ranged from the surface to $z=\sim 30 \mathrm{~km}$, with a vertical grid spacing of $\sim 500 \mathrm{~m}$ in the troposphere. D1 and D2 were integrated over $60 \mathrm{~h}$ (from 06:00 UTC, 8 August to 18:00 UTC, 10 August) and D3 was integrated over $54 \mathrm{~h}$ from 12:00 UTC, 8 August. Details in the simulation can be found in KC10. To avoid unrealistic signals while the vortex was spinning up and to focus on the rapid development and decay stages of Typhoon Saomai (2006), the results from the $48 \mathrm{~h}$ between 12:00 UTC, 8 August and 12:00 UTC, 10 August were selected and are presented in this study.

\section{Results}

\subsection{Domain-averaged HDTGW in the upper troposphere}

To examine the characteristics of upper-level divergence from the TGWs, HDTGW is calculated at each vertical level,

$$
\mathrm{HDTGW}=\left(\frac{\partial u^{\prime}}{\partial x}+\frac{\partial v^{\prime}}{\partial y}\right)_{\mathrm{GW}},
$$

where $u^{\prime}$ and $v^{\prime}$ are the perturbations of zonal and meridional winds from the domain-averaged zonal $(U)$ and meridional $(V)$ winds, respectively, which satisfy the frequency range of
IGWs:

$f^{2}<\hat{\omega}^{2}<N^{2}$,

where $f$ is the Coriolis parameter, $\hat{\omega}(=\omega-U k-V l)$ the intrinsic frequency, $\omega$ the ground-based frequency, $k$ and $l$ the zonal and meridional wavenumbers, respectively, and $N$ the Brunt-Väisälä frequency. The total horizontal divergence that will be shown in Fig. 2c is calculated from Eq.(1) without extracting IGW components. Note that total divergence is also calculated using the wind perturbations rather than total wind at model grid.

One of the interesting findings reported in $\mathrm{KC} 11$ is that the domain-averaged HDTGW in D3 showed alternating positive and negative signs with a period of about $18 \mathrm{~h}$. Although no spectral analysis of the HDTGW was performed in KC11, this result is somewhat unexpected because the dominant spectral peak of the TGWs shown in the vertical velocity of $\mathrm{KC} 10$ was less than $1 \mathrm{~h}$. In order to examine this discrepancy, we performed spectral analyses of the HDTGW with respect to time and space.

Figure 1a shows time-height cross section of the domainaveraged HDTGW in D3 from 12 to $18 \mathrm{~km}$ above ground level (a.g.1.) (Fig. 1a). The time series of the minimum sea level pressure (SLP), domain-averaged total divergence, domain-averaged HDTGW at $16 \mathrm{~km}$ a.g.l. (Fig. 1b), and domain-averaged vertical velocity averaged from 3 to $15 \mathrm{~km}$ a.g.l., and domain-averaged $30 \mathrm{~min}$ accumulated precipitation amount (Fig. 1c) are also included. Shown in Fig. 1e is the power spectral density (PSD) of HDTGW with respect to frequency at several selected heights. Figure 1a reveals that HDTGW in the upper troposphere show a periodic divergence and convergence pattern, especially above $13.5 \mathrm{~km}$ a.g.l. The magnitude of the divergence and convergence is larger when the typhoon is intensifying (from 17:00 UTC, 8 August to 17:00 UTC, 9 August), because intense convection accompanying the typhoon generates large amplitude gravity waves.

The domain-averaged total divergence (Fig. 1b) is positive in most times except for few hours near 20:00 UTC, 9 August at which the minimum SLP starts to increase. There is a significant correlation (coefficient of 0.72 at 0 lag with $99 \%$ confidence level) between the total divergence and HDTGW throughout the $36 \mathrm{~h}$ period (from 12:00 UTC, 8 August to 00:00 UTC, 10 August), before a decaying period. During a rapidly developing $12 \mathrm{~h}$ period (00:00 to 12:00 UTC, 9 August) in which the minimum SLP drops from 966 to $938.5 \mathrm{hPa}$, the total divergence and HDTGW are strongly correlated. During this period, IGWs contribute to the total divergence about $30 \%$ in UTLS. In this period, there is also a strong correlation between the minimum SLP and HDTGW as well as the minimum SLP and total divergence at $16 \mathrm{~km}$ a.g.l. The horizontal divergence/convergence that is vertically integrated from the surface to top of the atmosphere (model top in this case) can determine the surface pressure tendency in a hydrostatic atmosphere (Holton, 


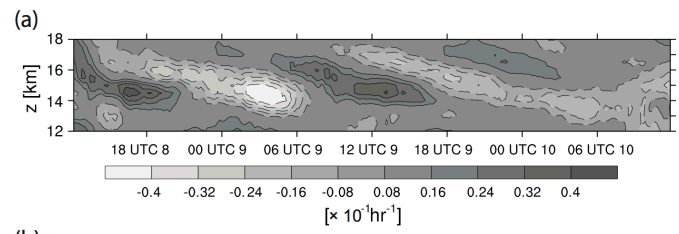

(b)

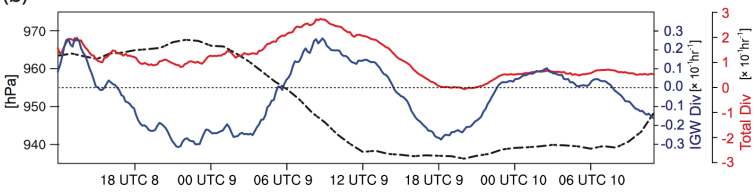

(c)
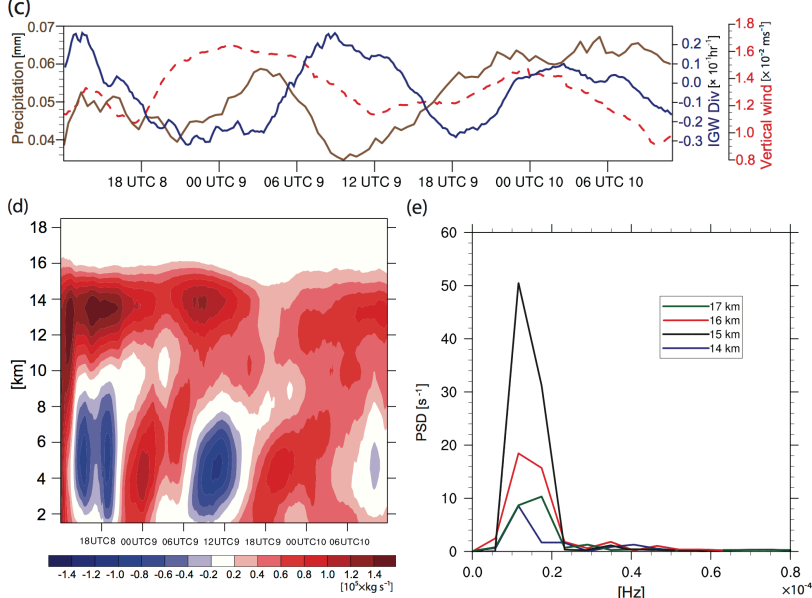

Fig. 1. (a) Time-height cross section of the domain-averaged HDTGW from 12 to $18 \mathrm{~km}$ a.g.l. Dashed and solid lines denote negative (convergence) and positive (divergence) values, respectively. (b) Time series of total divergence at $16 \mathrm{~km}$ a.g.l. (red), HDTGW (blue) and minimum sea-level pressure (dot dashed). (c) Time series of HDTGW at $16 \mathrm{~km}$ a.g.l. (blue), domain-averaged vertical velocity averaged over 3 to $15 \mathrm{~km}$ a.g.l. (red dashed), and domainaveraged $30 \mathrm{~min}$ accumulated precipitation amount (brown). (d) Time-height cross section of the vertical mass transport. (e) The power spectral density (PSD) of the HDTGW with respect to frequency at selected heights from 14 to $17 \mathrm{~km}$ a.g.l.

1992). The time-height cross section of domain-averaged total divergence (not shown) denotes that the magnitude of the divergence in UTLS is much larger than that of the convergence/divergence in the troposphere, and thus temporal variation in the vertically integrated divergence/convergence is largely determined by that in the divergence in UTLS. Strong correlations between the total divergence and minimum SLP, HDTGW and minimum SLP, and total divergence and HDTGW in UTLS during the rapidly developing period demonstrate the contribution of IGWs to typhoon evolution. It is noteworthy that it may be difficult to find causalities between variables at each time, because a continuous feedback process takes place.

The domain-averaged vertical velocity averaged over 3$15 \mathrm{~km}$ a.g.l. and domain-averaged $30 \mathrm{~min}$ accumulated precipitation amount (Fig. 1c) is generally well matched during $12 \mathrm{~h}$ of period of Typhoon intensification (00:00 UTC,
9 August to 12:00 UTC, 9 August), while they are less correlated in the last $24 \mathrm{~h}$ including a decaying period after 00:00 UTC, 10 August. Due to the interaction of typhoon and topography, the vertical wind and precipitation increase after 12:00 UTC, 9 August and produce another peak around 00:00 UTC, 10 August when outer rainbands reach Taiwan, as shown recently by Jang and Chun (2013). Correlation between the vertical velocity and HDTGW at $16 \mathrm{~km}$ a.g.l. varies with time. Before 17:00 UTC, 8 August and after 17:00 UTC, 9 August, there is a positive relation between the two variables, while in the developing period (17:00 UTC, 8 August17:00 UTC, 9 August), there is a clear negative correlation, most significantly during the rapidly developing period near 01:00 UTC, 9 August-12:00 UTC, 9 August.

The strong negative correlation between the two variables during the rapidly developing period is somewhat unexpected. In order to understand this result, we calculated timeheight cross section of the vertical mass transport (Fig. 1d) that is the sum of the upward mass transport and downward mass transport in D3, which are the domain sum of the positive and negative $\rho w$ (where $\rho$ is the air density and $w$ is the vertical velocity) in each grid, respectively. It shows that the total divergence and HDTGW at $16 \mathrm{~km}$ a.g.l. are positively correlated with the vertical transport in UTLS (9$16 \mathrm{~km}$ a.g.l.), while they are negatively correlated with the vertical transport below $9 \mathrm{~km}$. When we check the upward mass transport and downward mass transport separately, it is found that significant upward mass transport exists in the entire troposphere during the rapidly developing period, while strong downward transport exists at the same time in the lower layer. Considering that the D3 domain covers a rather wide area $(1191 \mathrm{~km} \times 1191 \mathrm{~km})$, as shown in Fig. 2, the downward motions occupied in relatively wider areas outside of the eyewall in the lower troposphere can contribute to the negative domain-averaged vertical velocity, while upward motions occupied in relatively wider areas of anvil clouds in the upper troposphere contribute to the positive domainaveraged vertical velocity in UTLS. The correlation between the HDTGW and precipitation is generally similar to that between the HDTGW and vertical velocity.

The PSD of HDTGW (Fig. 1e) is largest at $15 \mathrm{~km}$ a.g.l., and decreases rapidly below and above $15 \mathrm{~km}$ a.g.l. Two distinct spectral peaks exist at $1.16 \times 10^{-5} \mathrm{~s}^{-1}$ (with a period of about $24 \mathrm{~h}$ ) and at $1.7 \times 10^{-5} \mathrm{~s}^{-1}$ (with a period of about $16 \mathrm{~h}$ ) for most heights. The two peaks satisfy a $95 \%$ confidence level. The best-track data of Typhoon Saomai from the Japan Meteorological Agency (JMA) show that Saomai propagated to the northwest during the early stage, but stayed on a west-northwest path during our analysis period (see Fig. 1 of KC10). The latitudinal change in the typhoon track during the analysis period is from 22 to $27^{\circ} \mathrm{N}$, and the corresponding inertial periods range from 32 to $26 \mathrm{~h}$. This suggests that the dominant spectral peak of the HDTGW shown in Fig. 1e is near the inertial frequency. 

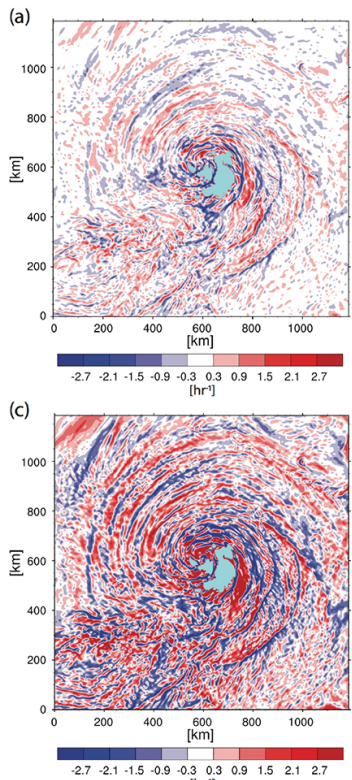

(e)

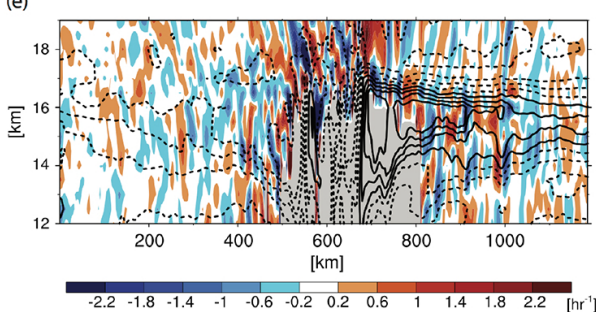

Fig. 2. (a) HDTGW at $16 \mathrm{~km}$ a.g.l., (b) radar reflectivity at $400 \mathrm{hPa}$, (c) total horizontal divergence at $16 \mathrm{~km}$ a.g.1. at 03:00 UTC, 9 August, and (d) phase difference between the total horizontal divergence and HDTGW shown in (a) and (c). In (d), gray and white regions denote in phase and out of phase between (a) and (c), respectively. Note that blue color denotes cloud-covering area in (a), (c), and (d). (e) $x-z$ cross section of zonal wind (contour) superimposed on HDTGW (shaded) at $y=600 \mathrm{~km}$ on 03:00 UTC, 9 August. Gray shaded region shows cloud-covering area. In (e), solid and dashed contours denote positive and negative values, respectively, with $6 \mathrm{~m} \mathrm{~s}^{-1}$ intervals from $-21 \mathrm{~m} \mathrm{~s}^{-1}$ to $21 \mathrm{~m} \mathrm{~s}^{-1}$.

Although the $24 \mathrm{~h}$ period in the HDTGW is within the frequency range of the IGWs, this variation could be related to diurnal effects, such as thermal tidal oscillation and daily rainfall variation. For the thermal tide to contribute to the present HDTGW variation, a semi-diurnal signal with even stronger power should also appear in the power spectrum of the HDTGW (Browner et al., 1977; Kossin, 2002; Yoshioka et al., 2005). However, the spectral powers near $12 \mathrm{~h}$ $\left(2.3 \times 10^{-5} \mathrm{~s}^{-1}\right)$ are nearly at a minimum for all heights (Fig. 1e). If the convective activity in Typhoon Saomai is associated with diurnal variation of precipitation, the solarsynchronous component can be linked to the HDTGW indirectly. In the present simulation, maximum precipitation occurred around 18:00 LST, which differs from the findings of a previous study by Jiang et al. (2011), who showed pre- cipitation maxima in the early morning and minima in the evening using 12 years of satellite data. Our analyses suggest that the $24 \mathrm{~h}$ period of the domain-averaged HDTGW is not likely to be related to thermal tidal oscillation or daily rainfall variation. From the Cryogenic Infrared Spectrometers and Telescopes for the Atmosphere (CRISTA) temperature data, Preusse (2001) found large-scale (about $3000 \mathrm{~km}$ wavelength) gravity waves near cloud top of Typhoon Winnie (1997) when the typhoon was intensifying. The study also showed a dominant period close to $24 \mathrm{~h}$ and the peak convective activity at 12:00 UTC (21:00 LST), outside of diurnal cycle.

\subsection{Spatial distribution of HDTGW}

In the previous section, we found that the dominant period of the domain-averaged HDTGW is $16-24 \mathrm{~h}$ and that it is strongly correlated with the evolution of a typhoon. This raises two questions: (i) why do the short-period wave components with the strongest powers, as shown in $\mathrm{KC10}$, disappear in the domain-averaging process, and (ii) what is the origin of the low-frequency components of domain-averaged HDTGW?

Figures $2 \mathrm{a}$ and $\mathrm{b}$ show a snapshot of the HDTGW field at $16 \mathrm{~km}$ a.g.l. and the model-produced radar reflectivity at $400 \mathrm{hPa}$ at 03:00 UTC, 9 August, respectively, when typhoon Saomai was rapidly developing. The HDTGW field has a complicated structure in each quadrant from the typhoon center, with an overall concentric shape, a spiral shape in the southeast (SE) with stronger amplitudes near the typhoon center propagating outward, and relatively small-scale features in the southwest (SW). This wavy pattern of the HDTGW represents convectively generated gravity waves that reach to $16 \mathrm{~km}$ a.g.l., consisting of high-frequency waves near the typhoon center and relatively low-frequency waves far from the typhoon center. The low-frequency spiral patterns are somewhat asymmetric, with stronger amplitudes east of the typhoon center. This asymmetric structure is likely related to the asymmetric convective activity of Typhoon Saomai before reaching its minimum SLP (at 12:00 UTC, 9 August), which was also reported recently by Jang and Chun (2013, Fig. 8). Unlike other regions, the HDTGW structure is complicated in the southwestern part of the typhoon center, which is because TGWs generated by smallscale convective clouds on the tails of a rainband (Fig. 2b) interfere with TGWs generated by the main convective clouds near the typhoon center. Note that $16 \mathrm{~km}$ a.g.l. is just above the cloud top in most regions, except for a few regions near the typhoon center where $16 \mathrm{~km}$ AGL is still inside the clouds.

In order to understand contribution of IGW to the total horizontal divergence in the upper troposphere, the horizontal divergence calculated using the total perturbation wind is shown in Fig. 2c. Note that HDTGW is the horizontal divergence calculated exclusively from IGW components 
that satisfy the dispersion relation of IGWs $\left(f^{2}<\hat{\omega}^{2}<N^{2}\right)$. Shown in Fig. $2 \mathrm{~d}$ is the phase between the total and gravitywave divergences. The values of +1 and -1 in Fig. $2 d$ correspond to in phase (same sign) and out of phase (different sign) between the divergence fields shown in Fig. 2a and c, respectively. Several interesting features can be seen from Fig. $2 \mathrm{c}$ and $\mathrm{d}$. First, the horizontal divergence by the total perturbation wind is generally similar to HDTGW, except that its magnitude is about 2-3 times larger in most regions. However, divergence (positive value) dominates convergence (negative value) in Fig. 2c, especially in the inner-core region, and consequently domain-averaged divergence is positive $\left(2.03 \times 10^{-5} \mathrm{~s}^{-1}\right)$, while domain-averaged HDTGW (Fig. 2a) is negative $\left(-2.7 \times 10^{-6} \mathrm{~s}^{-1}\right)$ at this specific time. Based on the time series of the domain-averaged total and gravity-wave horizontal divergences for the present case shown in Fig. 1b, total horizontal divergence also experiences periodic oscillation with the similar period of HDTGW, although it is always positive unlike HDTGW, and the maximum contribution of HDTGW to the total divergence is about $50 \%$ near 12:00 UTC, 9 August when the domain-averaged HDTGW becomes maximum, as shown in Fig. 1a. Second, the total and gravity-wave horizontal divergences are out of phase (white areas of Fig. 2d) mostly near the inner core and southeastern part of the typhoon center, while they are in phase in the southwestern and northwestern rainband regions. In order to understand contribution of relatively long wavelength components to the phase difference, we calculated the total divergence and HDTGW averaged over subdomains $(200 \mathrm{~km}$ by $200 \mathrm{~km})$ in the total domain shown in Fig. 2a. It is found (not shown) that there are more numbers of positive (divergence) subdomains in total divergence and of negative (convergence) subdomains in HDTGW, as consistent with Fig. 2a and c. Phase difference between the total divergence and HDTGW also reveals in phase in western subdomains and out of phase in the southern subdomains of inner core and south of the inner core. The out phase in the SE shown in Fig. 2d is not evident in the subdomain calculation. Considering that subdomain-averaged total divergence and HDTGW are due to relatively longerwavelength GWs, the phase calculated from the subdomainaveraged values is somewhat different from that shown in Fig. 2d, especially in SE where relatively short horizontal wavelength waves have strong powers, as will be shown in Fig. 3a. It is not clear at this moment what causes phase difference between the total and gravity-wave divergence fields, which need further investigation in the future.

While Typhoon Saomai was rapidly developing, a strong outflow layer developed near the tropopause. Figure $2 \mathrm{e}$ shows a $x-z$ cross section of the zonal wind (contour) superimposed on HDTGW (color) at the center of the typhoon $(y=600 \mathrm{~km})$ at 03:00 UTC, 9 August. HDTGW is large in a relatively wider region, about $\pm 300 \mathrm{~km}$, from the center $(x=600 \mathrm{~km})$ below about $17 \mathrm{~km}$ a.g.1., while waves with relatively long vertical wavelengths can propagate above (a)

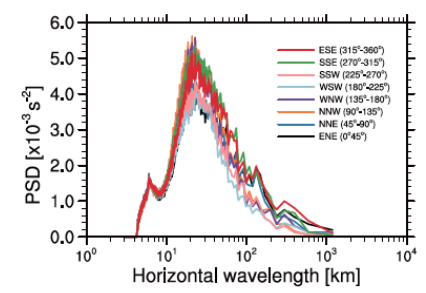

(b)

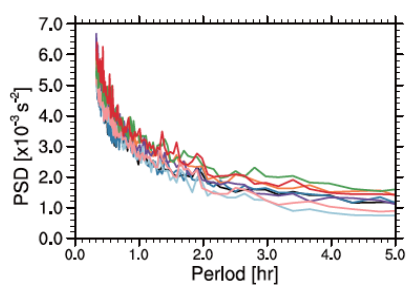

Fig. 3. PSDs of the HDTGW with respect to the (a) horizontal wavelength and (b) period in each propagation direction with $45^{\circ}$ intervals at $16 \mathrm{~km}$ a.g.l.

$17 \mathrm{~km}$ a.g.l., especially in the eastern part of typhoon center. This is likely because easterly background wind increasing with height enhances the vertical wavelengths of gravity waves.

Figure $2 \mathrm{a}$ and $\mathrm{b}$ show snapshots at specific times. In order to understand the general spectral characteristics of HDTGW, we calculated the PSD of a HDTGW with respect to the horizontal wavelength (Fig. 3a) and period (Fig. 3b) in each propagation direction with $45^{\circ}$ intervals at $16 \mathrm{~km}$ a.g.l. To do this, we compute the three-dimensional PSD of the HDTGW with respect to the zonal wavenumber $(k)$, meridional wavenumber $(l)$, and frequency $(\omega)$ at each altitude by fast Fourier transform (FFT). We then construct a PSD $(c, \varphi)$, where $c$ is the ground-based horizontal phase speed and $\varphi$ is the azimuthal direction, which are obtained by $c=\omega / \sqrt{k^{2}+l^{2}}$ and $\varphi=\cos ^{-1}\left(k / \sqrt{k^{2}+l^{2}}\right)$, respectively.

The horizontal wavelength spectrum (Fig. 3a) shows that dominant powers exist at wavelengths of $10-40 \mathrm{~km}$ with a peak near $20 \mathrm{~km}$ for all directions. The powers in the SE directions are relatively strong, whereas those in the SW directions are relatively weak for most wavelengths, with the exception that the strongest power for wavelengths between 10 and $20 \mathrm{~km}$ exists in the north-northwest (NNW: 90-135 ${ }^{\circ}$ ) direction. The period spectrum (Fig. 3b) shows that dominant powers exist at periods shorter than $1 \mathrm{~h}$, and similar to the wavelength spectrum, relatively strong powers exist in the SE directions and weak powers in the SW directions. The strong powers of HDTGW at horizontal wavelengths near $20 \mathrm{~km}$ and at periods shorter than $1 \mathrm{~h}$, as shown in Fig. $3 \mathrm{a}$ and $\mathrm{b}$, are somewhat expected, based on the vertical velocity fields reported by KC10. This suggests that high-frequency components of HDTGW have small horizontal scales, as shown in Fig. 2a, and that they tend to cancel when averaged over the domain.

\subsection{Sources of domain averaged HDTGW}

It was demonstrated in the previous section that the asymmetric structure of a typhoon can yield significant differences in the HDTGW within each quadrant, especially for highfrequency signals. However, low-frequency patterns are still present, even when higher-frequency signals are stronger in 


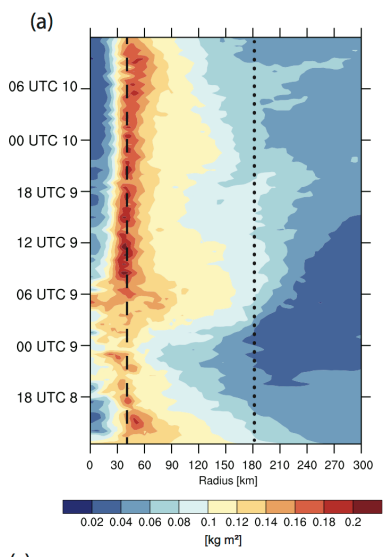

(c)

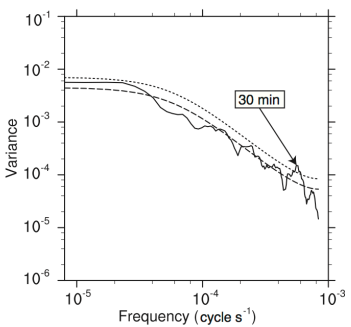

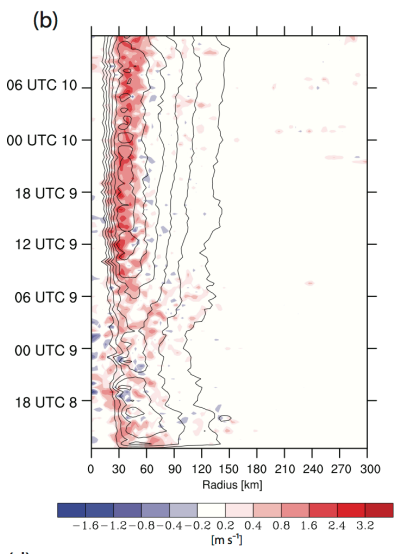

(d)

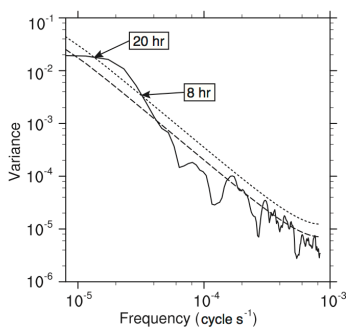

Fig. 4. (a) Hovmöller diagram of azimuthally averaged precipitable water (PW) in the simulation of typhoon Saomai. Dashed and dotted lines in (a) denote locations of spectral analyses performed in (c) and (d), respectively. (b) The same as in (a) except for the vertical velocity (color) at $1.5 \mathrm{~km}$ a.g.l. superimposed on the horizontal wind speed at $1.5 \mathrm{~km}$ a.g.1. from 25 to $50 \mathrm{~m} \mathrm{~s}^{-1}$ with $5 \mathrm{~m} \mathrm{~s}^{-1}$ intervals. The power spectrum of PW with respect to frequency are shown at (c) $40 \mathrm{~km}$ and (d) $180 \mathrm{~km}$ from the typhoon center. Dashed and dotted lines in (c) and (d) represent red noise and the $95 \%$ confidence level, respectively.

some regions, and the domain-averaged HDTGW in all four regions have a spectral peak at $24 \mathrm{~h}$ (not shown), as in Fig. 1a. To investigate the source of the low-frequency variation in HDTGW, we performed spectral analyses of the convective activity in two regions separately: the inner core region of stronger convective clouds and the outer rainband region of wider and intermittent convective clouds.

Figure 4a shows a Hovmöller diagram of azimuthally averaged precipitable water (PW) (Fig. 4a) and horizontal and vertical wind speed (Fig. 4b) from the Typhoon Saomai simulation and PSDs of PW with respect to frequency at $40 \mathrm{~km}$ (Fig. 4c) and $180 \mathrm{~km}$ (Fig. 4d) from the typhoon center. The PW contour in Fig. 4a is azimuthally averaged and represents the wavenumber 0 axisymmetric horizontal distribution of the PW from the typhoon center. Strong convective activity in the eyewall region is evidenced by a large PW value and wind speed near $30-60 \mathrm{~km}$, and is especially pronounced from 06:00 UTC, 9 August to 12:00 UTC, 10 August, with short-period fluctuations during that time. Note that there is a minimum SLP at 12:00 UTC, 9 August, and Saomai began to decay at 06:00 UTC, 10 August (Fig. 1b). Between

18:00 UTC, 8 August and 06:00 UTC, 9 August, the PW is rather small, not only near the eyewall but also in the rainband region. This is the time at which the domain-averaged HDTGW has a minimum value (convergence), as shown in Fig. 1a. It is not very clear at this moment whether this minimum value of HDTGW is related to wave activity or wave phase. Based on the time series of HDTGW and convective sources (vertical velocity and precipitation) shown in Fig. 1 and spatial structure of HDTGW shown in Fig. 2a, however, it is likely related to preferential wave phase in the D3 domain rather than wave activity.

The power spectrum of the PW at $40 \mathrm{~km}$ reveals spectral peaks at 26-30 min with a $95 \%$ confidence level (Fig. 4c). Outside of the eyewall region, the outward-propagating PW signal is well represented. This clearly exhibits that principal or secondary rainbands spiral periodically out from the center. The PSD of the PW in the rainband region (at $180 \mathrm{~km}$ ) has statistically significant spectral peaks at $8-20 \mathrm{~h}$ (Fig. 4d). This implies that the domain-averaged HDTGW is due largely to gravity waves generated by convective clouds in the rainbands with periods longer than $8 \mathrm{~h}$. Although largeamplitude gravity waves generated by vigorous convection near the eyewall with periods shorter than $1 \mathrm{~h}$ produce much stronger divergence and convergence simultaneously in the upper troposphere, they are for the most part cancelled out during the domain-averaging process.

As in the previous section, we divided the domain into an inner core and an outer band region. A radius of $150 \mathrm{~km}$ was chosen for the size of the inner core based on the PW and convective activities of Typhoon Saomai (Weatherford and Gray, 1988). Figure 5a presents the PSDs of HDTGW at $16 \mathrm{~km}$ a.g.l. with respect to the horizontal phase speed (radius) and propagation direction (azimuthal angle) in the total (left), inner core (middle), and outer rainbands (right) regions of D3. The HDTGW in the inner core region is isotropic, and the dominant powers are seen at phase speeds slower than $20 \mathrm{~m} \mathrm{~s}^{-1}$ and mostly at about $10 \mathrm{~m} \mathrm{~s}^{-1}$. The power of the HDTGW in the outer rainband region is much weaker, with relatively strong powers in the SE and NW directions. In the SE direction, notable powers extend to higher-phase speeds (up to $40 \mathrm{~m} \mathrm{~s}^{-1}$ ). The HDTGW PSD in the outer rainband region results from filtering of the TGWs by background winds in the UTLS, unlike in the inner core regions where convective clouds extend to almost $16 \mathrm{~km}$ a.g.l. and relatively highfrequency waves propagate vertically very quickly with less filtering by background wind. In the zonal wavenumber and frequency domain, the vertical propagation of IGWs is determined by two factors: latitude that determines the Coriolis parameter and the basic-state wind structure in the target height range of wave propagation (Chun et al., 2007). The domain-averaged zonal and meridional winds change from southeasterly to northeasterly below $16 \mathrm{~km}$ a.g.l. (see Fig. 4 of $\mathrm{KC10}$ ) during the time considered in the present study, which covers the azimuthal angles between 150 and $240^{\circ}$. Therefore, a large portion of TGWs in these azimuthal angles 
(a)

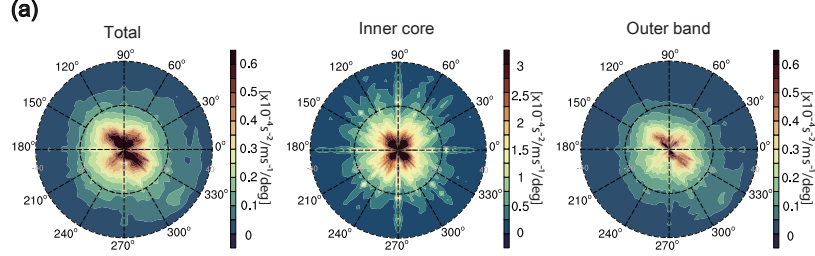

(b)

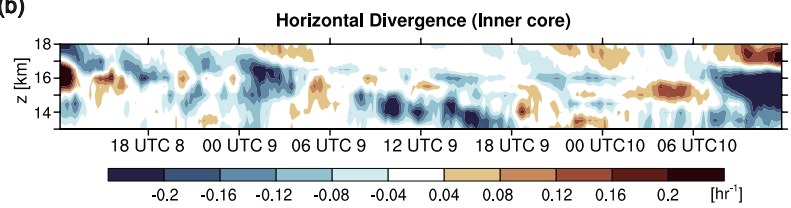

(c)

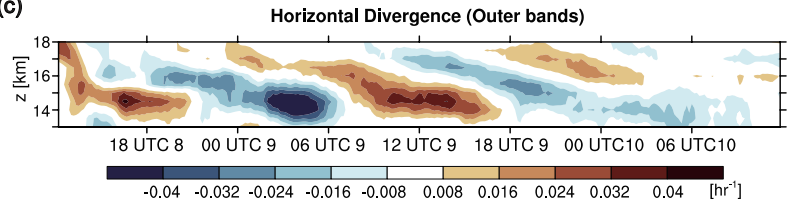

Fig. 5. (a) PSD of the HDTGW at $16 \mathrm{~km}$ a.g.l. with respect to the horizontal phase speed (radius) and propagation direction (azimuthal angle) in the total (left), inner core (middle), and outer rainbands (right) regions of D3. The inner core is defined by a $150 \mathrm{~km}$ radius from the typhoon center, and the outer rainband region is defined by a region subtracting the inner core region from D3. Two dashed rings in each circle in (a) represent phase speeds of 20 and $40 \mathrm{~m} \mathrm{~s}^{-1}$, respectively. Time-height cross sections of domainaveraged HDTGW in the inner core and outer rainband regions are shown in (b) and (c), respectively.

are filtered out at $16 \mathrm{~km}$ a.g.l. in the outer rainband region, as revealed by the relatively weak powers in the directions of $150-270^{\circ}$ in Fig. 5 a.

The time series of the domain-averaged HDTGW in the inner core (Fig. 5b) shows much more irregular temporal variations compared with the total (Fig. 1a) and outer bands (Fig. 5c), mainly due to irregular temporal variations in the convective activity of the inner core region. The HDTGW in the outer band region is strikingly similar to the total HDTGW shown in Fig. 1a. This result implies that, during the averaging process, high-frequency variation in the inner core region disappears, with only the divergence pattern in the outer rainband region remaining. The spectral analysis of the domain-averaged HDTGW at $16 \mathrm{~km}$ a.g.l. with respect to the frequency reveals (not shown) that PSDs in both the total and the outer band regions have two distinct peaks at 24 and $16 \mathrm{~h}$ periods. The inner core region has relatively strong powers at several high frequencies, including a primary peak at $16 \mathrm{~h}$ and a secondary peak at about $3.4 \mathrm{~h}$.

\section{Conclusions}

In this study, the characteristics of the HDTGW are examined using mesoscale simulation of typhoon Saomai and a spectral analysis method. It was found that HDTGW shows strong power in the lower stratosphere at horizontal wavelengths and periods of about $10-20 \mathrm{~km}$ and less than an hour, respectively, regardless of the varying azimuthal angle of wave propagation from the typhoon center. This is consistent with a previous study by $\mathrm{KC} 10$ of the vertical velocity field. However, the domain-averaged HDTGW in the typhoon center had a spectral peak at $24 \mathrm{~h}$. The $24 \mathrm{~h}$ period of the averaged HDTGW stems from the IGWs generated by the relatively weak and low-frequency convective clouds in the spiral rainbands, and showed no clear association with the thermal tides or the diurnal precipitation trend. The strong HDTGW powers in the inner core region with relatively short-period components are mostly filtered out during the domain-averaging process.

A time series of the domain-averaged HDTGW in the UTLS is well correlated with the total divergence in UTLS as well as the minimum SLP of Saomai, implying typhoon and gravity wave feedback through HDTGW. Note that the long and low-frequency GWs contribute mostly to the domainaveraged HDTGW and total divergence, while short and high-frequency GWs are mostly filtered out during the average process. Given that the upper-level divergence is known to have a crucial role in changes in typhoon intensity, TGWs and, more specifically, HDTGW in the UTLS should be considered in future typhoon intensity studies, and especially in the outer rainband region, as one of the mechanisms used to explain the temporal variation in the minimum sea-level pressure of typhoon centers. In the present study, we have considered a feedback between TGWs and typhoon evolution through the horizontal divergence. This feedback process can be possible for other types of convective systems and convective GWs. In addition, other feasible ways of representing the feedback process with variables other than horizontal divergence in UTLS should be deduced, which requires further studies on this topic.

Acknowledgements. This work was supported by the National Research Foundation of Korea (NRF) grant funded by the Korean government (MSIP) (no. 20110000060 and 2013R1A2A2A01014644).

Edited by: T. J. Dunkerton 


\section{References}

Bosart, L. F., Bracken, W. E., Molinari, J., Velden, C. S., and Black, P. G.: Environmental influences on the rapid intensification of Hurricane Opal (1995) over the Gulf of Mexico, Mon. Weather Rev., 128, 322-352, 2000.

Browner, S. P., Woodley, W. L., and Griffith, C. G.: Diurnal oscillation of the area of cloudiness associated with tropical storms, Mon. Weather Rev., 105, 856-864, 1977.

Chun, H.-Y., Goh, J.-S., and Kim, Y.-H.: Characteristics of inertiogravity waves revealed in rawinsonde data observed in Korea during 20 August to 5 September 2002, J. Geophys. Res., 112, D16108, doi:10.1029/2006JD008348, 2007.

Dhaka, S. K., Takahashi, M., Shibagaki, Y., Yamanaka, M. D., and Fukao, S.: Gravity wave generation in the lower stratosphere due to passage of the typhoon 9426 (Orchid) observed by the MU radar at Shigaraki $(34.858 \mathrm{~N}, 136.108 \mathrm{E})$, J. Geophys. Res., 108, 4595, doi:10.1029/2003JD003489, 2003.

Emanuel, A. K.: An air-sea theory for tropical cyclones. part I: Steady-state maintenance, J. Atmos. Sci., 43, 585-604, 1986.

Holton, J. R.: An introduction to dynamic meteorology, Academic press, San Diego, USA, 1992.

Jang, W. and Chun, H.-Y.: The effects of topography on the evolution of typhoon Saomai (2006) under the influence of tropical storm Bopha (2006), Mon. Weather Rev., 141, 468-489, 2013.

Jiang, H., Liu, C., and Zipser, E. J.: A TRMM-based tropical cyclone cloud and precipitation feature database, J. Appl. Meteor. Climatol., 50, 1255-1274, 2011.

Kim, S.-Y. and Chun, H.-Y.: Stratospheric gravity waves generated by Typhoon Saomai (2006): Numerical modeling in a moving frame following the typhoon, J. Atmos. Sci., 67, 3617-3636, doi:10.1175/2010JAS3374.1, 2010.
Kim, S.-Y. and Chun, H.-Y.: Impact of typhoon-generated gravity waves in the typhoon development, Geophys. Res. Lett., 38, L01806, doi:10.1029/2010GL045719, 2011,

Kim, S.-Y., Chun, H.-Y., and Baik, J.-J.: A numerical study of gravity waves induced by convection associated with Typhoon Rusa, Geophys. Res. Lett., 32, L24816, doi:10.1029/2005GL024662, 2005.

Kossin, J. P.: Daily hurricane variability inferred from GOES infrared imagery, Mon. Weather Rev., 130, 2260-2270, 2002.

Kuester, M. A., Alexander, M. J., and Ray, E. A.: A model study of gravity waves over Hurricane Humberto (2001), J. Atmos. Sci., 65, 3231-3246, 2008.

Merrill, R. T.: Environmental influences on hurricane intensification, J. Atmos. Sci., 45, 1678-1687, 1988.

Preusse, P.: Satellitenmessungen von schwerewellen in der mittleren atmosphäre mit CRISTA, Dissertation, Bergische Universität, available at: http://elpub.bib.uni-wuppertal.de/ servlets/DerivateServlet/Derivate-412/d080111.pdf (last access: 25 March 2014), 2001.

Rappin, E. D., Morgan, M. C., and Tripoli, G. J.: The impact of outflow environment on tropical cyclone intensification and structure, J. Atmos. Sci., 68, 177-194, 2011.

Weatherford, C. L. and Gray, W. M.: Typhoon structure as revealed by aircraft reconnaissance. Part I: Data analysis and climatology, Mon. Weather Rev., 116, 1032-1043, 1988.

Yoshioka, M. K., Kurihara, Y., and Ohfuchi, W.: Effect of the thermal tidal oscillation of the atmosphere on tropical cyclones, Geophys. Res. Lett., 32, L16802, doi:10.1029/2005GL022716, 2005. 